\title{
Does exposure to radiation emitted from mobile jammers influence the spatial memory?
}

\author{
A. Shahi', F. Shahnazar², S. Nematolahi², A. Dehghan4, \\ M.B. Shojaeifard $5,6^{*}$
}

\author{
${ }^{1}$ Lab Sciences Student, School of Medicine, Fasa University of Medical Sciences, Fasa, Iran \\ ${ }_{2}^{2}$ Medical Student, School of Medicine, Fasa University of Medical Sciences, Fasa, Iran \\ ${ }_{3}^{3}$ Biostatics Department, School of Medicine, Shiraz University of Medical Sciences, Shiraz, Iran \\ ${ }^{4}$ Non Communicable Diseases Research Center, School of Medicine, Fasa University of Medical Sciences, Fasa, Iran \\ ${ }^{5}$ Physiology Department, Fasa University of Medical Sciences, Fasa, Iran \\ ${ }^{6}$ Ionizing and non-ionizing radiation protection research center, Shiraz University of Medical Sciences, Shiraz, Iran
}

\section{- Original article}

*Corresponding author:

Manzar B. Shojaeifard, Ph.D., E-mail: shojaeim@sums.ac.ir

Revised: July 2020

Accepted: August 2020

Int. J. Radiat. Res., October 2021; 19(4): 993-1000

DOI: $10.29242 /$ ijrr.19.4.993

\begin{abstract}
Background: The central nervous system is sensitive to radiation exposure as environmental pollution. This project aimed to evaluate the influence of jammer exposure radiation on spatial learning and memory. Materials and Methods: 50 adult male Sprague-Dawley rats were divided into five groups. The experimental groups were exposed to jammer radiation for $2 \mathrm{~h} /$ day once or $2 \mathrm{~h} /$ day for two weeks. Sham groups were similar to the experimental groups which were exposed to switch-off. The distance from the jammer router to the animals' cages was $30 \mathrm{~cm}$. In the second phase, using Morris Water Maze, the effect of jammer exposure radiation on spatial learning and memory was studied. Results: Data showed that radiation exposure once a day for $2 \mathrm{~h}$ caused a significant increase in the learning procedure in the experimental group1, but we observed an increase in the parameters of distance and escape latency in finding the platform for two weeks' exposure in the experimental group 2. Conclusion: Results indicated that probably the motivation of escape and use of the different cues led to learning in the animals, while disrupting mobile performance through jammer router from animal environment caused a better brain performance in the spatial learning and memory in short term jammer radiation-treated animals group. However, with the same test conditions, in long term jammer the radiation-treated animals' motivation reduced, which affected the responses and performance and reduced the learning. Moreover, environmental conflicts such as radiofrequency waves lead to behavioral alternations.
\end{abstract}

Keywords: Jammer router, Morris Water Maze, spatial learning and memory.

\section{INTRODUCTION}

Electromagnetic waves, as the fourth source of environmental pollution, induce various biological effects in different organs (1). The mobile phone can be considered as a widely used device; hence, anybody can call anytime, and this makes some problems and troubles for others. To solve this problem, mobile jammer devices are used extensively as a signal blocking system. This system prevents radio waves transferring by sending noise (radiofrequency waves with the same frequency as the mobile phone), and as a result, it interferes with the signal transmission. Therefore, mobile phones will be disabled. Today, jammer systems are used in different places, such as libraries, seminar rooms, hospitals, temples, etc. The range of the radius in which jammers can disable the mobile phone depends on the mobile jammer model (2).

For all organisms, learning ability is 
necessary, particularly because they need to get food and so on to leave their home and nest, etc. This capacity is vital for them, without this, they could not survive (3).

Learning is data acquisition and data storage, but memory is persistent storage and retention of learned information (4). Most of our knowledge and skills are not inherited and they are learned from our environment. Learning is obtaining new information from the environment surrounding us and memory is remembering and renewing of that knowledge over time (5). Synaptic plasticity involves the first step of learning and memory formation. Also, memory is the storage skill of the experiences and remembering the learned processes or events (6).

Many complications may happen as the result of memory loss in the central nervous system. Some steps of memory storage last from a few seconds until one minute. This is called short term memory or active memory. Temporary information is stored in the memory for a short period (up to a minute) $(7,8)$; if it takes a longer time (up to several years or even life period), it is named long term memory $(7,8)$. The short-term and long-term memories have a difference in duration and capacity of what is being stored (9). Morris water maze(MWM which was purchased from Azma Tecknique company, Tabriz, Iran.) is an instrument used for evaluation of the spatial memory and learning capabilities of the rodents (10). Rodents are innate swimmers; however, they have the tendency to stay outside water.

Hence, swimming induced motivation for the animal to actively seek a place within the pool. The rats with this technique learn by swimming to find the platform. Therefore, the animal actively seeks the platform situation, and uses the available spatial, proximal and distal cues in its environment. (3) In the MWM method, rats try to learn the connecting link between the cue and place for finding the escape platform. This technique that involves four trails/day with a short time in each trail (120 seconds) allows the animal to gain good spatial learning within a short time (4-6 days) ${ }^{(3,10)}$. The aim of this study was to evaluate the mobile jammer radiation effects on the spatial learning and memory in adult male rats.

Various studies have been conducted on the exposure to $2.45 \mathrm{GHz} \mathrm{MW}$ radiation, showing controversial results on the learning and memory performance. Therefore, this research through evaluation of the jammer radiation effects on the learning and memory performance using MWM test as active avoidance test would bridge these research gaps. Important point in this project is that we used mobile jamer router as a signal blocking system, but pervious research mainly concentrated on the mobile/ WiFi radition effects on the learning and memory.

\section{MATERIAL AND METHODS}

\section{Animals}

In the present study, 50 mature male Sprague -Dawley rats (200-250 g, and age about 6-7 weeks) were housed in a temperaturecontrolled room $\left(25 \pm 1^{\circ} \mathrm{C}\right)$ and $12 \mathrm{~h}$ light/dark cycle (light on at 7:00 a.m.) with free access to water and food (standard rodent chow) all the time. Before the experiment, all the rats were adapted to the new environment/housing about one week. All experimental procedures as well as housing conditions were in accordance with international principles of (European Council Directive 86/609/EEC of 24 November 1986) about animals' treatment.

\section{Experimental design}

Rats were randomly divided into 5 groups $(\mathrm{N}=10)$. For the control group there was no intervention, the sham groups 1 and 2 included the rats exposed to switch-off instrument for $2 \mathrm{~h}$ once in a day or $2 \mathrm{~h} /$ day for two weeks, respectively. Jammer router was located at 30 $\mathrm{cm}$ distance from the rats' cages. Thus, jammer router was arranged in the center of the circle and the rat cages were in the circle perimeter in a distance of $30 \mathrm{~cm}$ from the center. Experimental groups $1 \& 2$ included the animals exposed to a switched-on mobile jammer system once a day for $2 \mathrm{~h}$ or $2 \mathrm{~h} /$ day for two weeks in sequential order.

Int. J. Radiat. Res., Vol. 19 No. 4, October 2021 


\section{Exposure set up}

The animals were placed in Plexiglas cages individually and exposed to radiation. The electromagnetic field measurements were done using an Aaronia Spectran HF-4060 device (Euscheid, Germany) with the frequency range $100 \mathrm{MHz}-6 \mathrm{GHz}$. The frequency band, power output, and power density measured from electromagnetic fields of Jammer router were 1052-1979(MHz),-32 \pm 3 (dB mw), 86.18000 $(\mu \mathrm{w} / \mathrm{m} 2)$, and ICNIRP $0.31(\%)$ respectively. Jammer router (MB06Mobile Blocker) works in four different frequency ranges (Global System for Mobile Communications [GSM], digital cellular service, code division multiple access, third generation). Shielding radii for this jammer are up to $40 \mathrm{~m}$.

\section{Behavioral Study-Morris Water Maze}

MWM test usually assesses the spatial memory and learning via recording of escape latency and distance. Briefly, MWM consists of a round pool. MWM (purchased from Azma Tecknique company, Tabriz, Iran.) is comprised of a large black circular water tank $(130 \mathrm{~cm}$ in diameter, $50 \mathrm{~cm}$ in height). Water pool is filled up with tap water and it should be used as the maze; two hypothetically principal axes bisect the maze perpendicular to each other and to create four positions of North, South, East and West.

In this way, dividing the maze produces four quadrants/areas equally. In other words, four cardinal points of the hypothetical compass directions (N, E, S, and $\mathrm{W}$ ) are formed. In this phase, learning experiment began through MWM set up. The training for the first step of Morris water maze test with platform was performed one day after exposure radiation phase. This step was called the acquisition phase. The rats were handled for 4 days; in the start position, they were individually dropped in the water pool at one of the four quadrant /cardinal points of the hypothetical compass directions (quadrant NW, quadrant NE, quadrant SW, quadrant SE). Rats from one trial to the others were dropped in the water pool in a quasi-random sequence (11).

The location of the metal platform (diameter/surface: $10 \mathrm{~cm}$ ) was in the middle of quadrant 4 or SE. Initially, the platform was above the water surface $(1 \mathrm{~cm})$ and it was visible for rats. When the animals found it, they rested on the platform for 20s. If the rat could not find the platform within $60 \mathrm{~s}$, it was led toward the platform, and allowed to rest for $20 \mathrm{sec}$. Then, it was returned to the cage. Each rat repeated this procedure for 4 trials; however, the drop area of a rat in the water tank was different. Also, the break time between the trails was $1 \mathrm{~min}$.

This procedure was repeated on days 2, 3, 4, but in these steps the platform was hidden $1 \mathrm{~cm}$ below the water. A learning test or Probe trial (without platform) was performed on day 5. The rat was dropped into the water pool from the furthest place relative to the hidden platform place. The video tracking system of MWM was set up to monitor the pathway of the rat swimming. This system records how long it takes for the rat to find the platform and stay on it. Also, the system records the duration of the presence of the rats in each quadrant as well as the distance and velocity spent in each zone by each rat. When the rat lay on the platform, tracking system stopped recording (12)., This is to inform you that for carrying out this project on animals, we acted exactly based on all the principles of research on animals issued by International Council for Laboratory Animal Science (ICLAS) 1986 in UK and 2010 /63/in EU and also, ethics committee of Fasa University of Medical Sciences. The Ethics Committee approved this project with the. Ethics Code: IR.FUMS.REC.1394.4

\section{Statistical analysis}

All data are reported via means \pm SEM. Statistical differences among groups were determined by analysis of variance (ANOVA) and Tukey's post hoc tests, using SPSS software (ver. 15; SPSS, Inc., Chicago, IL, USA). P-values of $<0.05$ were considered statistically significant. 


\section{RESULTS}

I-Effects of short term (once, 2h/day) exposure to mobile jammer radiation on the spatial learning in rats.

The influence of mobile jammer radiation on the spatial learning and memory was examined; all rats during training learned to locate the platform.

\section{A-Parameter of traveled distance}

Learning is indicated by reducing the swimming path or the length of travel distance for finding the hidden platform in the quadrant SE. Statistical analysis of the mean of the length of traveled distance or swim path (table 1) showed a significant decrease during the trial phase on the 5 th day in experiment 1 compared with the sham 1 group $(\mathrm{P}=0.001)$ in traveled distance from the area quadrant NE to quadrant SE, as well as swim path inside the quadrant SE. The values in the control and experiment 1 groups were statistically similar.
Furthermore, the mean of the total distance (the sum of traveled distance from different areas such as quadrant 1 to quadrant SE plus swim path from quadrant NE to hidden platform in quadrant SE and traveled distance from quadrant SW to quadrant SE plus swim path inside the quadrant SE showed a significant decrease between the experiment 1 and the sham1 group $(\mathrm{P}=0.004)$.

\section{B-Parameter of escape latency}

Learning is determined through time reduction, the time it takes the animal to find the platform (latency period) in the designed zone. (table 2), One way ANOVA showed a significant decrease in the mean of escape latency (table 2) for finding the hidden platform in quadrant SE, during quadrant NE (the time it takes the animal to reach to quadrant SE from zone SE) in experiment 1 compared with the sham 1 group $(\mathrm{P}=0.034)$. The values of escape latency in the control and experiment 1 groups in all quadrants separately and totally were statistically similar.

Table 1. The effect of jammer exposure radiation for $2 \mathrm{~h} /$ day in the distance that rats moved to find the platform in the Morris water maze.

\begin{tabular}{|c|c|c|c|c|c|}
\hline Groups & Quadrant NW [cm] & Quadrant NE [cm] & Quadrant SW [cm] & $\begin{array}{c}\text { Quadrant SE [cm] } \\
\text { (hidden platform) }\end{array}$ & $\begin{array}{c}\text { Total distance to } \\
\text { hidden platform }\end{array}$ \\
\hline Control & $163.43 \pm 32.96$ & $241.187 \pm 32.1$ & $279.06 \pm 41.25$ & $160.47 \pm 28.28$ & $813.68 \pm 115.13$ \\
\hline Sham1 & $292.14 \pm 23.15$ & $319.23 \pm 23.64$ & $494.5 \pm 29.10$ & $353.86 \pm 28.09$ & $1349.47 \pm 94.36$ \\
\hline Experiment1 & $223.37 \pm 22.02$ & $200.03 \pm 20.85^{*}$ & $370.37 \pm 28.9$ & $220.44 \pm 22.22^{*}$ & $964.25 \pm 73.98^{*}$ \\
\hline
\end{tabular}

Values are expressed as Mean \pm SEM $(n=10)$. *Significant difference with sham $1,(P=0.001, P=0,001, P=0.004$ respectively).

Table 2. Effect of jammer radiation for $2 \mathrm{~h} /$ day once in escape latency to the platform in the Morris water maze.

\begin{tabular}{|c|c|c|c|c|c|}
\hline Groups & Quadrant NW Sec & Quadrant NE Sec & Quadrant SW Sec & $\begin{array}{c}\text { Quadrant SE } \\
\text { (hidden platform) }\end{array}$ & $\begin{array}{c}\text { Total latency to } \\
\text { the platform }\end{array}$ \\
\hline Control & $6.99 \pm 1.56$ & $11.23 \pm 1.92$ & $22.10 \pm 2.85$ & $7.71 \pm 1.44$ & $46.692 \pm 6.09$ \\
\hline Sham1 & $15.22 \pm 1.18$ & $15.59 \pm 1.28$ & $33.94 \pm 2.62$ & $17.97 \pm 1.25$ & $79.41 \pm 3.97 \mathrm{ne}$ \\
\hline Experiment1 & $12.357 \pm 1.34$ & $11.24 \pm 1.13^{*}$ & $30.67 \pm 2.41$ & $15.07 \pm 1.96$ & $66.08 \pm 5.05$ \\
\hline
\end{tabular}

Values are expressed as Mean \pm SEM $(n=10)$. * Significant difference with sham $1, P=0.034$.

II-Effects of long term (2h/day for two weeks) exposure to mobile jammer radiation on the spatial learning in rats.

\section{A-Parameter of traveled distance}

Tables 3 and 4 summarize the results of jammer radiation exposure for $2 \mathrm{~h} /$ day during two weeks on the animal's spatial memory. The results showed the mean of traveled distance or swim pathway (table 3) for finding the hidden platform in quadrant SE, revealing a significant increase during the trial phase on the 5th day in experiment 2 compared with the sham 2 and control groups in all quadrants separately(the traveled distance from each quadrant1/NW, NE, SW, or SE separately) for finding the platform in quadrant SE and totally (the sum of the swim

Int. J. Radiat. Res., Vol. 19 No. 4, October 2021 
path from each quadrant NW, NE, SW, or SE for climbing on the platform $(\mathrm{P}=0.001)$. The values in the control and the sham 2 group were statistically similar in all zones. In this part of the experiment, we found that exposure to jammer radiation for two weeks impaired learning owing to the results showing an increase in the traveled distance or swim pathway for finding the hidden platform. This is in conflict with the learning definition.

\section{B-Parameter of escape latency}

Statistical analysis on the mean of escape latency (table 4) for finding the hidden platform in quadrant 4 showed a significant increase during the trial phase on the 5th day in experiment 2 compared with the sham 2 and control groups in all quadrants separately (the time spent from each quadrant NW, NE, SW or SE to quadrant SE separately) and totally (the sum of the time taken from quadrant NW, NE, SW or SE to quadrant $\mathrm{SE}$ to reach quadrant $\mathrm{SE}$ ) $(\mathrm{P}=0.001)$. The values in the control and the sham 2 groups were statistically similar in all zones. Given that learning was determined through time reduction, these data showed the opposite this issue.

Table 3. The effect of jammer radiation $2 \mathrm{~h} /$ day for two weeks on the distance needed to find the platform in the Morris water maze.

\begin{tabular}{|c|c|c|c|c|c|}
\hline Groups & Quadrant NW[cm] & Quadrant NE [cm] & Quadrant SW [cm] & $\begin{array}{l}\text { Quadrant SE [cm] } \\
\text { (hidden platform) }\end{array}$ & $\begin{array}{c}\text { Total distance to } \\
\text { hidden platform }\end{array}$ \\
\hline Control & $163.43 \pm 32.94$ & $241.19 \pm 32.11$ & $279.06 \pm 41.25$ & $160.47 \pm 28.28$ & $813.68 \pm 115.13$ \\
\hline Sham2 & $163.44 \pm 19.04$ & $241.19 \pm 23.83$ & $208.67 \pm 25.79$ & $155.94 \pm 24.51$ & $626.875 \pm 73.66$ \\
\hline Experiment2 & $302.22 \pm 25.3^{*} 3$ & $344.69 \pm 24.34^{*}$ & $426.65 \pm 27.51^{*}$ & $343.35 \pm 23.87^{*}$ & $1352.32 \pm 76.81^{*}$ \\
\hline
\end{tabular}

Values are expressed as Mean \pm SEM $(n=10)$. ${ }^{*}$ Significant difference with sham 2 in all quadrant separately and total quadrant $(P=0.001$ respectively).

Table 4. The effect of jammer radiation $2 \mathrm{~h} /$ day for two weeks on the escape latency for finding the platform in the Morris water maze.

\begin{tabular}{|c|c|c|c|c|c|}
\hline Groups & Quadrant NW (sec) & Quadrant NE (sec) & Quadrant SW (sec) & $\begin{array}{c}\text { Quadrant SE (hidden } \\
\text { platform) (sec) }\end{array}$ & $\begin{array}{c}\text { Total latency to } \\
\text { the Platform }\end{array}$ \\
\hline Control & $6.52 \pm 1.52$ & $11.23 \pm 1.92$ & $22.10 \pm 2.85$ & $7.23 \pm 1.43$ & $46.69 \pm 6.09 \mathrm{Sec}$ \\
\hline Sham2 & $7.05 \pm 1.08$ & $11.03 \pm 1.53$ & $18.04 \pm 2.09$ & $8.50 \pm 1.66$ & $42.43 \pm 4.85 \mathrm{Sec}$ \\
\hline Experiment2 & $17.60 \pm 1.53^{*}$ & $17.11 \pm 1.18^{*}$ & $29.94 \pm 2.11^{*}$ & $19.30 \pm 1.49 *$ & $80.33 \pm 3.75 \mathrm{Sec}$ \\
\hline
\end{tabular}

Values are expressed as Mean \pm SEM $(n=10) *$ Significant difference with control and sham2 ( $p=0.001$ respectively).

\section{DISCUSSION}

In this study, we used the Morris water maze technique to evaluate the influence of short term and long term exposure to jammer radiation on the spatial memory in rats. The results showed that once a day for $2 \mathrm{~h}$ exposure to the jammer radiation caused a significant increase in the learning procedure of the Morris water maze (in jammer radiation-treated animals' experimental group 1), but we observed an increase in the parameters of distance (swim path length) and escape latency in finding the hidden platform for two weeks' exposure to the jammer radiation (in jammer radiation-treated animals experimental group 2). Our results indicated that probably in groups of exposure to jammer radiation once a Int. J. Radiat. Res., Vol. 19 No. 4, October 2021 day for $2 \mathrm{~h}$ the motivation of escape from the water, and the use of different cues (outside of the water pool) provided better performance learning in these animals.

However, the long term exposure process probably have provided adaptation with water and different cues (outside of the water pool) in the animal's environment, so the escape motivation has reduced. Moreover, change in motivation influences the response and performance, which affects the learning (14) Also, effortful learning depends on performancerelated feedback (15) On the other hand, learning requires practice through repetition which usually undermines the motivation. (16) These results and those of other research studies show that impaired memory following exposure to 
EMF radiation are in this line. Hence, disrupting mobile performance through jammer router from the animal's environment couldn't result in better brain performance on the spatial memory and learning in jammer radiation-treated animals experimental 2 for a subsequent two weeks'-exposure.

Various studies show that restudying the same material or repeating the same work can reduce long-term retention and knowledge acquisition (17). On the other hand, cognitive neuroscience explains that learning level depends on dopamine neurotransmitter release (18) since dopamine increases the association of between stimulus and response, and develops target behavior level such as learning, memory, and motor performance. RF-EMF exposure decreases the dopamine concentration in the striatum. (The striatum is an area in the forebrain which affects the cognition processes). RF-EMF exposure leads to reduction in the number and size of the synaptic vesicles in the striatal presynaptic nerve endings. (19) Also, neuropsychological researches explain performance-contingent reward in a test or performance pressure-induced test, providing a reduction in long term memory ${ }^{20)}$.

The sensitivity of CNS to environmental conflicts such as radiofrequency waves causes behavioral alternations; therefore, a change in behavior leads to alternation in the activity of the nervous system(21). Lahijani et al. (2011) observed histological changes after exposure of EMF in the brain of chicks (22).

In 2012, it was revealed that mobile phone radiation-induced impairments in MWM performance (23). Also, the results of animal studies have been demonstrated that radiation is disrupting spatial learning and memory performance via Morris water maze technique. Frangopoulos et al. (2010) for $2 \mathrm{hr}$./day during a period of 4 days, Megha et al. (2012), for $2 \mathrm{hr}$./ day during a 30-day period as well as Ntzouni et al. (2013) for $90 \mathrm{~min} /$ day during 66-148 day period showed spatial memory deficit. $(24,25,26)$.

However, Haghani et al. (2013) did not observe any behavioral effect on the spatial memory after $15 \mathrm{~min} /$ day during an 8 or 24-week exposure duration (27). On the other hand, maybe irradiation led to an increase in the reactive oxygen species (ROS) level(28, 29), ROS effects on the cognitive behavior, and impairs spatial memory $(25,28,30)$. The current results obtained from EMF researchers are inconsistent; some of them reported radiofrequency waves emitted from a cell phone could stimulate ROS production in some different tissues including neural tissue. It was also reported that ROS production after RF radiation exposure was induced in neuroblastoma cells (91), in SN56 cells (32) probably, because of electrical activities in the neural tissue and the nearness of cell phone to head during communication; hence, the C.N.S will be the favored target (32). Although the intensity of RF-EMFs is very low, the oscillatory frequencies correspond to some of the oscillation frequencies recorded in the neuronal tissue and could interfere with neuronal activity (33).

Moreover, another research reported RF-EMFs emitted by cell phones were absorbed in the brain within a range that could influence the neuronal activity (34,35).The research findings of increased metabolic activities in the closest regions to the antenna during acute radiation exposure to cell phone suggest that brain RF-EMFs absorption probably improve the brain tissue excitability (36).

One study indicated 50-minute cell phone exposure was associated with increased brain glucose metabolism in the region closest to the antenna (37) .One of these studies reported CBF decreases in the region with maximal RF-EMF exposure (38). In line with this, our research center observed the radiation emitted from mobile jammer router reduced fasting blood glucose level(39). Besides, long-term exposure radiation effects emitted by mobile Jammer on reproduction parameters in rats are different based on the life stage (neonate, immature and mature)(40). Exposure of mature rats to mobile phone jammer radiation led to significant differences in RBC, hematocrit and hemoglobin, MCHC, MCV, RDWCV, and platelets compared to the control group, but immature rats didn't show significant effects on these factors (41).

In a way, learning about the results of one's

Int. J. Radiat. Res., Vol. 19 No. 4, October 2021 
actions requires feedback, which can modify the learner's behavior. Motivation for doing work probably-influences the performance feedback and effectively improves learning (42). It has been suggested that Jammer router may lead to better brain function and improvement in the spatial memory and learning in experimental groups compared to other groups.

\section{CONCLUSION}

Our results indicated that probably in short term jammer radiation-treated animals group, the motivation of escape from the water and the use of the different cues (outside of the water pool) accompanied by blocking the transfer of signals and disrupting mobile radiation provided better learning. However, in long term jammer, radiation-treated animals group repeating the same work (test) undermines the motivation; this influences the neural responses and the performance, and then reduces the long-term retention and knowledge acquisition. Moreover, environmental conflicts such as radiofrequency waves lead to behavioral alternations; however, disrupting mobile performance through jammer router from the animal's environment couldn't improve the brain performance on the spatial memory and learning.

\section{ACKNOWLEDGEMENTS}

The authors of the present study sincerely thank the Vice President of research and the staff of Research Fasa University of Medical Sciences for their support. The authors wish to thank Dr. Shokrpour of English Department, Shiraz University of Medical Sciences, Shiraz, Iran, for his invaluable assistance in editing this manuscript.

\section{Financial Disclosure}

The authors of the present study declare that none of them have any kinds of "Conflict of Interest" (such as financial, personal, political, or academic) that would potentially affect their judgment.

\section{Funding/Support}

The present study was financially supported by Fasa University of Medical Sciences, Fasa, Iran.

Conflicts of interest: Declared none.

\section{REFERENCES}

1. Levitt BB and Lai H (2010) Biological effects from exposure to electromagnetic radiation emitted by cell tower base stations and other antenna arrays. Environmental Reviews, 18: 369-395.

2. Mahato SK and Vimala C (2014) Cellular signals jamming system in $2 \mathrm{G}$ and 3G. In International Conference on Signal Processing, Embedding System and Communication Technologies and Their Applications for Sustainable and Renewable Energy, 3: 242-247.

3. Vorhees CV and Williams MT (2014) Assessing spatial learning and memory in rodents. ILAR Journal, 55(2): 310332.

4. Okano, H, Hirano, T, Balaban E (2000) Learning and memory. Proceedings of the National Academy of Sciences, 97(23): 12403-12404.

5. Kandel ER, Dudai Y, Mayford MR (2014) The molecular and systems biology of memory. Cell, 157(1): 163-186.

6. Takeuchi T, Duszkiewicz AJ, Morris RG (2014) The synaptic plasticity and memory hypothesis: encoding, storage and persistence. Philosophical Transactions of the Royal Society B: Biological Sciences, 369(1633): 201302.

7. Baddeley AD (1992) Working Memory. Science, Vol. 255, No. 5044, pp. 556-559. Doi: 10.1126/. 5044,

8. Quillfeldt JA (2016) Behavioral methods to study learning and memory in rats. Springer, Cham. In Rodent Model as Tools in Ethical Biomedical Research (pp. 271-311)

9. Cowan N (2008) What are the differences between longterm, short-term, and working memory? Progress in Brain Research, 169: 323-33.

10. Barnhart CD, Yang D, Lein PJ (2015) Using the Morris water maze to assess spatial learning and memory in weanling mice. PloS one, 10(4): e0124521.

11. Jadidi M, Firouzabadi S, Rashidipour A, Bolouri B, Sajadi A (2009) Does whole body exposure to GSM-950 MHz electromagnetic fields affect acquisition and consolidation of spatial information in rats? Int J Radiat Res, 7(1): 57-62.

12. Morris R (1984) Developments of a water-maze procedure for studying spatial learning in the rat. Journal of neuroscience methods, 11(1): 47-60.

13. Brandeis R, Brandys Y, Yehuda S (1989) The use of the Morris water maze in the study of memory and learning. International Journal of Neuroscience, 48(1-2): 29-69.

14. Dickerson KC and Adcock RA (2018) Motivation and Memory. Stevens' Handbook of Experimental Psychology and Cognitive Neuroscience, 1: 1-36.

15. DePasque $S$ and Tricomi E (2015) Effects of intrinsic moti- 
vation on feedback processing during learning. Neurolmage, 119: 175-18.

16. Kim SI (2013) Neuroscientific model of motivational process. Front Psychol, 4: 98.

17. Kuhbandner C, Aslan A, Emmerdinger K, Murayama K (2016) Providing extrinsic reward for test performance undermines long-term memory acquisition. Frontiers in psychology, 7: 79.

18. Montague PR, Dayan P, Sejnowski TJ (1996) A framework for mesencephalic dopamine systems based on predictive Hebbian learning. Journal of Neuroscience, 16(5): 19361947.

19. Kim JH, Lee CH, Kim HG, Kim HR (2019) Decreased dopamine in striatum and difficult locomotor recovery from MPTP insult after exposure to radiofrequency electromagnetic fields. Scientific Reports, 9(1): 1-13.

20. Hinze SR and Rapp DN (2014) Retrieval (sometimes) enhances learning: Performance pressure reduces the benefits of retrieval practice. Applied Cognitive Psychology, 28 (4): 597-606.

21. Lai H (1999) Memory and Behavior. The Biological Effects, Health Consequences and S tanda rds for Pulsed Radiofrequency Field", Sicily, Italy. An international seminar sponsored by the International Commission on Nonionizing Radiation Protection and the World Health Organization, at the Ettoll Majorare, Centre for Scientific Culture, Erice, Sicily, Italy.

22. Lahijani MS, Farivar S, Khodaeian M (2011) Effects of $50 \mathrm{~Hz}$ electromagnetic fields on the histology, apoptosis, and expression of c-Fos and $\beta$-catenin on the livers of preincubated white Leghorn chicken embryos. Electromagnetic Biology and Medicine, 30(3): 158-169.

23. Li Y, Shi C, Lu G, Xu Q, Liu S (2012) Effects of electromagnetic radiation on spatial memory and synapses in rat hippocampal CA1. Neural Regeneration Research, 7(16): 1248.

24. Fragopoulou AF, Miltiadous $P$, Stamatakis A, Stylianopoulou F, Koussoulakos SL, Margaritis LH (2010) Whole body exposure with GSM $900 \mathrm{MHz}$ affects spatial memory in mice. Pathophysiology, 17(3): 179-187.

25. Megha K, Deshmukh PS, Banerjee BD, Tripathi AK, Abegaonkar MP (2012) Microwave radiation induced oxidative stress, cognitive impairment and inflammation in brain of Fischer rats. Indian J Exp Biol, 50(12): 889-96.

26. Ntzouni MP, Skouroliakou A, Kostomitsopoulos N, Margaritis LH (2013) Transient and cumulative memory impairments induced by GSM $1.8 \mathrm{GHz}$ cell phone signal in a mouse model. Electromagnetic Biology and Medicine, 32 (1): 95-120.

27. Haghani M, Shabani M, Moazzami K (2013) Maternal mobile phone exposure adversely affects the electrophysiological properties of Purkinje neurons in rat offspring. Neuroscience, 250: 588-598.

28. Yurekli Al, Ozkan M, Kalkan T, Saybasili H, Tuncel $H$, Atukeren P, Seker S (2006) GSM base station electromagnetic radiation and oxidative stress in rats. Electromagnetic Biology and Medicine, 25(3): 177188.

29. Azimzadeh M, Jelodar GA, Namazi F, Soleimani F (2018) Exposure to radiofrequency wave (RFW) generated by a base transceiver stations (BTS) antenna model affects learning and memory in female more than male rats. Int $\mathrm{J}$ Radiat Res, 16(4): 487-491.

30. Sokolovic D, Djindjic B, Nikolic J, Bjelakovic G, Pavlovic D, Kocic G, Pavlovic V (2008) Melatonin reduces oxidative stress induced by chronic exposure of microwave radiation from mobile phones in rat brain. Journal of Radiation Research, 49(6): 579-586.

31. Luukkonen J, Hakulinen P, Mäki-Paakkanen J, Juutilainen J, Naarala J (2009) Enhancement of chemically induced reactive oxygen species production and DNA damage in human SH-SY5Y neuroblastoma cells by $872 \mathrm{MHz}$ radiofrequency radiation. Mutation Research/Fundamental and Molecular Mechanisms of Mutagenesis, 662(1-2): 54-58.

32. Del Vecchio G, Giuliani A, Fernandez M, Mesirca P, Bersani F, Pinto R, Calza L (2009) Effect of radiofrequency electromagnetic field exposure on in vitro models of neurodegenerative disease. Bioelectromagnetics: Journal of the Bioelectromagnetics Society, the Society for Physical Regulation in Biology and Medicine, the European Bioelectromagnetics Association, 30(7): 564-572.

33. Kim JH, Yu DH, Huh YH, Lee EH, Kim HG, Kim HR (2017) Long-term exposure to $835 \mathrm{MHz}$ RF-EMF induces hyperactivity, autophagy and demyelination in the cortical neurons of mice. Scientific Reports, 7(1): 1-12.

34. Hyland GJ (2000) Physics and biology of mobile telephony. The Lancet, 356(9244), 1833-1836.

35. Schonborn F., Burkhardt M, Kuster N (1998). Differences in energy absorption between heads of adults and children in the near field of sources. Health Physics, 74(2): 160-168.

36. Kleinlogel $H$, Dierks $T$, Koenig $T$, Lehmann $H$, Minder A, Berz R (2008) Effects of weak mobile phoneelectromagnetic fields (GSM, UMTS) on event related potentials and cognitive functions. Bioelectromagnetics: Journal of the Bioelectromagnetics Society, the Society for Physical Regulation in Biology and Medicine, the European Bioelectromagnetics Association, 29(6): 488-497.

37. Volkow ND, Tomasi D, Wang GJ, Vaska P, Fowler JS, Telang F, Wong C (2011) Effects of cell phone radiofrequency signal exposure on brain glucose metabolism. Jama, 305 (8): 808-813.

38. Aalto $S$, Haarala $C$, Brück $A$, Sipilä $H$, Hämäläinen $H$, Rinne JO (2006) Mobile phone affects cerebral blood flow in humans. Journal of Cerebral Blood Flow \& Metabolism, 26 (7): 885-890.

39. Shooli FS, Mortazavi SAR, Jarideh S, Nematollahii S, Yousefi F, Haghani M,Shojaei-Fard MB (2016) Short-Term exposure to electromagnetic fields generated by mobile phone jammers decreases the fasting blood sugar in adult male rats. Journal of Biomedical Physics \& Engineering, 6(1): 27.

40. Owjfard M, Fard SM, Tamadon A (2015) Effects of LongTerm Exposure to Radiofrequency Radiations Emitted by Common Mobile Jammers on Sperm Quality Parameters in Rat. International Journal of Fertility \& Sterility, 9: 46.

41. Shojaeifard MB, Jarideh $S$, Owjfard $M$, Nematollahii $S$, Talaei-Khozani T, Malekzadeh M (2018) Electromagnetic fields of mobile phone jammer exposure on blood factors in rats. Journal of Biomedical Physics \& Engineering, 8(4): 403.

42. DePasque S and Tricomi E (2015) Effects of intrinsic motivation on feedback processing during learning. Neurolmage, 119: 175-186.

Int. J. Radiat. Res., Vol. 19 No. 4, October 2021 